\title{
A CLINICAL STUDY ON ADVERSE DRUG REACTIONS OF COMBINATION THERAPY OF DAPAGLIFLOZIN AND METFORMIN IN THE TREATMENT OF TYPE 2 DIABETES MELLITUS
}

\author{
SYED SAFIULLAH GHORI*, AMIT KAPOOR \\ Department of Hospital and Clinical Pharmacy, Anwar-Ul-Uloom College of Pharmacy, New Mallepally, Hyderabad, Telangana, India. \\ Email: safiullahghori@gmail.com
}

Received: 28 November 2016, Revised and Accepted: 28 December 2016

\begin{abstract}
Objective: This study aimed at determination of safety and efficacy of combination therapy of dapagliflozin and metformin in the treatment of Type 2 diabetes mellitus.

Methods: A total of 50 patients were enrolled in the study depending on demographic parameters and clinical data of the patients. The primary efficacy criterion was the change in glycated hemoglobin (HbA1c) after a minimum of 12 weeks of treatment. Secondary efficacy parameters were HbA1c value after 12 weeks, fasting and 1 hrs postprandial glucose, serum insulin and triglyceride levels, after a standardized meal, all after 12 weeks of treatment. Safety and tolerability were evaluated by the incidence of adverse events reported by patients. Patient visits to the clinical center were scheduled at screening, start of the run-in period.
\end{abstract}

Results: Reductions in levels of postprandial plasma glucose were observed in all the active treatment groups. The reductions in patients receiving metformin plus dapagliflozin combination therapy were significantly greater $(\mathrm{p}<0.0001)$. It was clear that lower postprandial plasma insulin levels despite higher postprandial plasma glucose levels suggest decreased $\beta$-cell function. Changes in fasting serum insulin observed from baseline to the end of treatment did not differ significantly between metformin plus dapagliflozin combination therapy and metformin monotherapy and showed no consistent trend.

Conclusion: The results from the study suggest that the combination of the drugs was effective in controlling glycemic levels and also were safe. No serious adverse drug reactions were reported by the patients when used daily once for 6 months.

Keywords: Dapagliflozin, Metformin, Postprandial and glycemic.

(C) 2017 The Authors. Published by Innovare Academic Sciences Pvt Ltd. This is an open access article under the CC BY license (http://creativecommons. org/licenses/by/4. 0/) DOI: http://dx.doi.org/10.22159/ajpcr.2017.v10i3.16380

\section{INTRODUCTION}

Diabetes mellitus is a major global health problem and an increasing cause of morbidity and mortality [1]. The term diabetes mellitus describes a metabolic disorder of multiple etiology, characterized by chronic hyperglycemia with disturbances of carbohydrate, fat and protein metabolism resulting from defects in insulin secretion, insulin action or both [2]. The diseased state underlying the diagnosis of diabetes mellitus are classified into four categories namely Type 1 - insulin-dependent diabetes mellitus, Type 2 - noninsulindependent diabetes mellitus, and Type 3 - gestational diabetes mellitus, and others. According to recent estimates, approximately 285 million people worldwide $(6.6 \%)$ in the $20-79$ year age group have diabetes and by 2030, 438 million people $(7.8 \%)$ of the adult population, is expected to have diabetes [3].

The World Health Organization estimates that worldwide, there are currently 220 million people living with diabetes [4]. In 2010, there were 45.2 million cases of Type 2 diabetes mellitus (T2DM) in India. Of these, 14.7 million and 30.5 million were found in rural and urban areas, respectively. By the end of 2011 and 2020, data monitoring estimates that the total prevalent cases of Type 2 diabetes increased to 47.2 and 69.7 million, respectively [5]. The major cities of India (New Delhi, Mumbai, Kolkata, Chennai, Bangalore, Hyderabad, and Ahmadabad) have got an estimated 7.3 million prevalent cases of Type 2 diabetes in 2010. Dapagliflozin is a sodium-glucose cotransporter 2 (SGLT2) inhibitor which prevents glucose reabsorption in the kidney [6]. It is indicated in the management of T2DM, and functions to improve glycemic control in adults when combined with diet and exercise. Metformin lowers blood glucose levels by various mechanisms such as suppressing hepatic gluconeogenesis and glucose output from liver, enhancing insulin mediated glucose disposal in muscle and fat, retarding intestinal absorption of glucose, other hexoses, amino acids and vitamin-B12 and interfering with mitochondrial respiratory chain [7]. This study was conducted to determine the safety and efficacy of combination of these two drugs in patients with T2DM.

\section{MATERIALS AND METHODS}

\section{Materials}

Combination of dapagliflozin $5 \mathrm{mg}$ plus metformin $500 \mathrm{mg}$ was used in the study The patients which were included in the study were male or female, aged between 30 and 75, who had been diagnosed with T2DM for at least 1 year and whose diabetes was inadequately controlled by diet and metformin taken at a stable dose for at least 3 months. Furthermore, the patients were required to have glycated hemoglobin $(\mathrm{HbA} 1 \mathrm{c}), \geq 7.5 \%$ and $\leq 10.5 \%$ at baseline, body mass index between 23 and $40 \mathrm{~kg} / \mathrm{m}^{2}$, and stable body weight ( $5 \%$ ) over the 3 months preceding enrolment. Subjects with serious medical conditions including hepatic or renal dysfunction, decompensate heart failure, serious hemopoietic disorders, or any gastrointestinal disorders were excluded from the study [8]. Patients who used either any oral antidiabetics other than metformin within the 30 days before enrolment or diuretics and glucocorticoids unless at a constant dose in the 3 months before enrolment were not included.

\section{Methodology}

The study was conducted in Prime Hospital, Hyderabad. An ethical clearance, ECR/381/Inst/AP/2013 was obtained from the hospital and all patients were requested to fill the informed consent form. Around 50 patients, who satisfied the eligibility criteria, were included 
during the study period. These patients were evaluated according to the treatment protocol. Data collection form was designed to collect data of the patients including personal details (name, age, sex height, weight, address, marital status, and occupation) family history, and past medical history, complications, and life style modification [9].

Primarily a detailed history of the patients enrolled was ascertained and entered in the case report form. Furthermore, a detailed previous history of drugs such as antibiotics and any history of previous hospitalization, associated illness and habits and diet any significant family history was also recorded. Patient visits to the clinical center were scheduled at screening, start of the run-in period. HbA1c was measured at screening, baseline, week 12, and thereafter at each visit if the preceding visit's value had been $>11 \%$. HbA1c was measured by high performance liquid chromatography, serum glucose by the glucose-oxidase method [10], serum insulin by double antibody radioimmunoassay. Fasting blood glucose and fasting urine glucose was measured at screening and week 12 .

\section{Statistical analysis}

Statistical analysis was done using GraphPad Prism software to calculate the probability of significance.

\section{RESULTS}

A total of 50 patients were enrolled in the study. Considering the first parameter of gender there were 30 males and 20 females enrolled in the study group (Table 1). Average baseline HbA1c (\%) in reference group was 8.2 in test group (Graph 1). The change in the HbA1c (\%) of the patients who responded clinically is presented in the Graph 2. Table 2 and Graph 2 present the overall efficacy assessment after the drug therapy for 6 months. The change in the HbA1c (\%) was considered to measure the efficacy in both groups. The test group and reference group both showed clinical response. There was significant change in HbA1c (\%) after the drug treatment. The adverse drug reactions reported by the subjects of both groups were recorded (Table 3). Flatulence was reported by two subjects in reference and one in test. Diarrhea was reported by five in reference and two in the test group. Nausea was reported by one in reference and two in test group, abdominal cramps were not reported in the reference group but one subject experienced the condition. Constipation was reported by seven subjects in both reference and test groups. Back pain was reported by one in reference and two in test group presented in Graph 3. Hypotension was reported by five patients, lowering of both systolic and diastolic BP in patients. It was clear that lower postprandial plasma insulin levels despite higher postprandial plasma glucose levels suggest decreased $\beta$-cell function. Changes in fasting serum insulin observed from baseline to the end of treatment

Table 1: Baseline demographic parameters

\begin{tabular}{ll}
\hline Parameters & Test group $\mathbf{n}=\mathbf{5 0}$ \\
\hline Age & $57.3 \pm 9.0$ \\
Weight & $91.0 \pm 15.5$ \\
Males & 30 \\
Females & 20 \\
BMI & 31.1 \\
Duration of disease & 7.2 \\
HbA1c $(\%)$ & 8.2 \\
\hline
\end{tabular}

BMI: Body mass index, HbA1c: Glycated hemoglobin

Table 2: Change in HbA1c (\%) EOT

\begin{tabular}{ll}
\hline Change in HbA1c (\%) & Test group $(\mathbf{n = 5 0})$ \\
\hline 6.5 & 29 \\
6.8 & 11 \\
7.2 & 5 \\
7.5 & 5 \\
\hline
\end{tabular}

EOT: End of treatment, HbA1c: Glycated hemoglobin did not differ significantly between metformin plus dapagliflozin combination therapy and metformin monotherapy and showed no consistent trend.

\section{DISCUSSION}

Dapagliflozin, one of group SGLT2 inhibitors, is effective as monotherapy and in combination with other antihyperglycemic agents in reducing HbA1C in T2DM [11]. This study demonstrates that dapagliflozin provides a greater clinical response or effect of strong glycemic control, as indicated by the marked reductions in $\mathrm{HbA1c}$ and plasma glucose levels in middle-aged patients in whom Type 2 diabetes is insufficiently controlled by dietary management. The reductions in fasting and postprandial plasma glucose as well as in HbA1c in response to combination of dapagliflozin plus metformin therapy. The Test drug acts synergistically to confer this additional glycemic control, especially on postprandial plasma glucose peaks, and may thereby help to reduce the risk of microvascular and macrovascular diabetic complications. From our study, the 3-6 months treatment showed greater efficacy and safety for short-term study in the finished population. The hypotension observed in the study might be due to combination therapy.

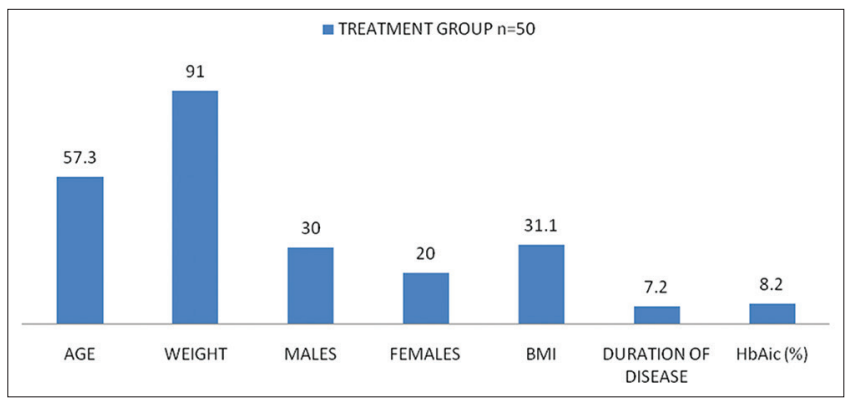

Graph 1: Baseline demographic characters

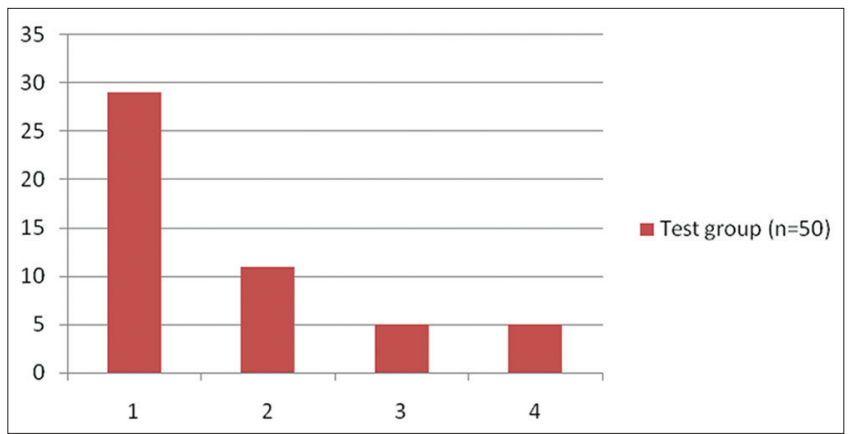

Graph 2: Change in glycated hemoglobin (\%) end of treatment

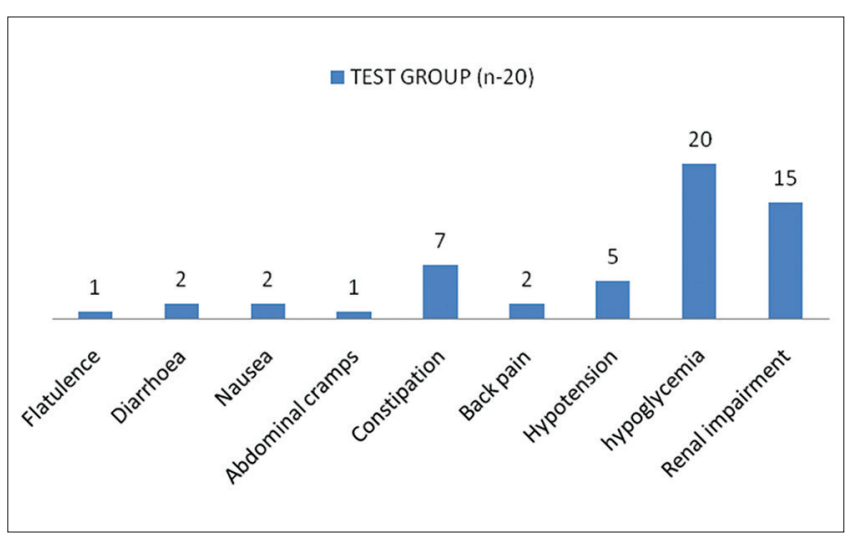

Graph 3: Adverse drug reactions reported 
Table 3: Adverse drug reactions

\begin{tabular}{ll}
\hline Adverse drug reactions & Test group (n-20) \\
\hline Flatulence & 1 \\
Diarrhea & 2 \\
Nausea & 2 \\
Abdominal cramps & 1 \\
Constipation & 7 \\
Back pain & 2 \\
Hypotension & 5 \\
Hypoglycemia & 20 \\
Renal impairment & 15 \\
\hline
\end{tabular}

T2DM is a prevalent, progressive disease with a need for innovative therapeutic agents to continue to advance disease management. The decrease in insulin resistance has been shown to have effects on lipid profiles as patients who develop T2DM [12]. Besides $\beta$-cell failure, the major pathophysiological event contributing to the development of T2DM is the resistance of target tissues to insulin [13]. Dapagliflozin is the second agent in a new class of oral antihyperglycemic drugs: SGLT2 inhibitors. SGLT2 is responsible for the majority of renal glucose reuptake; inhibition of the cotransporter allows for increased renal glucose excretion that consequently leads to reduced plasma glucose levels [14]. Because this mechanism does not require the action of insulin, dapagliflozin rarely causes hypoglycemia and is effective in patients both early and late in the course of their disease. Studies of dapagliflozin have demonstrated efficacy both as monotherapy and in combination with oral antihyperglycemic agents and insulin. The patients responded to the combination of drugs dapagliflozin a new SGLT2 drug has shown superior clinical response in reducing the blood glucose level, the clinical response was significantly more in the test group. The results show that the drug is safe when studied for the period of 6 months, further studied must be done to check the safety of the drugs in long-term use. The combination of SGLT2 drug and metformin have shown significant efficacy in patients with T2DM for 6 months. The study suggests that the combination of these drugs have shown minimal adverse drug reactions when used for the periods of 6 months.

\section{CONCLUSION}

In conclusion, the results from the study suggest that the combination of the drugs were effective in controlling glycemic levels and also safe, no patient had reported serious adverse drug reactions when used daily once for 6 months. The study also suggests that the patients with hypotension may have risk for severe hypotension. The adverse drug reactions were studied for short-term duration. No patient have been reported any serious adverse drug reactions. The study suggests that the drug administration for 6 months is safe and effective. Further studies should be done on the combination of drugs for long term.

\section{ACKNOWLEDGMENT}

The authors are thankful to Dr. Arvind Patnaik, Syncorp Technologies Pvt. Limited, Vijeta Classic Empire, No 301, $3^{\text {rd }}$ Floor, beside ICICI Bank, Chaithanyapuri, Dilsukhnagar, Hyderabad - 500 060, Telangana for his support in the present research.

\section{REFERENCES}

1. Pan CY, Gao Y, Chen JW, Luo BY, Fu ZZ, Lu JM, et al. Efficacy of acarbose in Chinese subjects with impaired glucose tolerance. Diabetes Res Clin Pract 2003;61(3):183-90.

2. Alberti KG, Zimmer PZ. Definition, diagnosis and classification of diabetes mellitus and its complications. Part 1: Diagnosis and classification of diabetes mellitus, provisional report of a WHO consultation. Diabet Med 1998;15:539-53.

3. Martha S, Kennedy N. Pancreatic hormones and anti diabetic drugs. In: Basic and Clinical Pharmacology. Vol. 9. New York, NY: McGraw-Hill Co.; 2004. p. 693-715.

4. Rang HP, Dale MM. The endocrine pancrease and the control of blood glucose in pharmacology. Vol. 5. Edinburgh: Churchill Livingstone; 2003. p. $380-93$

5. Jermendy G. Can type 2 diabetes mellitus beconsider preventable? Diabetes Res Clin Pract 2004;68:73-81.

6. Powers AC. Diabetes mellitus. In: Harrison's Principle of Internal Medicine. Vol. 16: 2001. p. 2114-5

7. IDF. Diabetes Atlas. $4^{\text {th }}$ ed. Belgium: International Diabetes Federation; 2009.

8. Markus RU. Alpha glucosidase inhibitor in thetreatment of diabetes mellitus. In: Diabetes Mellitus: A Fundamental and Clinical Text. Vol. 3. Philadelphia, PA: Lippincott Williams \& Wilkins; 2004. p. 115-1159.

9. Hanefeld M, Cagatay M, Petrowitsch T, Neuser D, Petzinna D, Rupp M. Acarbose reduces the risk for myocardial infarction in type 2 diabetic patients: Meta analysis of seven long term studies. Eur Heart J 2004;24(1):10-6.

10. Basevi V, Di Mario S, Morciano C, Nonino F, Magrini N. Comment on: American Diabetes Association. Standards of medical care in diabetes--2011. Diabetes Care 2011;34 Suppl 1:S11-61.

11. John R, Buse B. Management of type 2 diabetes mellitus in cecil. Essentials Med 2001;5:1231-46.

12. Nayak BS, Bhaktha G. Inconsistent lipid profiles exhibited among the diabetic Asian Indians of India and Trinidad - A comparative study. Int J Pharm Pharm Sci 2016;8(12):60-3.

13. Ritonga RH, Suprapti B, Khatib J. The influence of sodium orthovanadate on p85 and gsk-3 expressions to the blood glucose regulation of type 2 diabetic mice (Mus musculus) model. Int J Pharm Pharm Sci 2015;7(1):115-9.

14. Howard BV, Howard WJ. Pathophysiology and treatment of lipid disorders indiabetes. Diabetes Mellitus 2005;14: 563-79. 